\title{
Advances in intrauterine technique training
}

\author{
Anne Connolly, ${ }^{1}$ Sarah Rybowski ${ }^{2}$
}

\author{
'General Practitioner with \\ Special Interest in Gynaecology, \\ The Ridge Medical Practice, \\ Bradford, UK \\ ${ }^{2}$ Global Medical Affairs \\ Physician, Bayer HealthCare \\ Pharmaceuticals, Berlin, \\ Germany \section{Anne Connolly, \\ The Ridge Medical Practice, Bradford BD7 3JX, UK; anne.connolly@bradford.nhs.uk} \\ Correspondence to \\ Received 28 July 2011 \\ Accepted 23 September 2011 \\ Published Online First \\ 15 November 2011
}

\section{Background}

Virtual reality for practical training has come a long way since the early days of flight simulation during the first part of the last century. It is used in many environments, including spatial awareness, military and surgical training. Threedimensional physical models (generally plastic) and computer-aided two-dimensional training allow participants to interact visually with objects but usually lack tactile sensation feedback. One improvement is to incorporate haptic technology. By definition, haptics (from the Greek word haptikos, pertaining to the sense of touch and pronounced hap-tiks) is a tactile feedback technology that takes advantage of a user's sense of touch. Stimulation of the user's sense of touch is accomplished by applying forces, vibrations, resistances and/or motions back to the user by means of special input/output devices called 'end effectors' (jointed mechanical arm pieces), joysticks, data gloves or similar devices. Thus this is the science of applying touch (tactile) sensation and control to interactions within computer applications or graphics. In combination with a visual display, haptic technology can be used as an adjunct to training programmes for skilled tasks requiring handeye co-ordination.

\section{Haptic technology}

Haptic technology is gaining wider use in several industries, and it has been particularly successful in medicine during the last decade. ${ }^{1}$ Applications range from training in dental techniques ${ }^{1}$ and laparoscopic surgery ${ }^{2}$ to the rehabilitation of patients with cerebral palsy or stroke with functionally impaired upper or lower limbs. ${ }^{3}$ Recently, a group in Illinois, USA reported that they had used haptic technology successfully to assess the variation in surgical proficiency among orthopaedic residency trainees. ${ }^{5}$ A systematic review of the literature has suggested that simulator training for knee arthroscopy employing haptic technology and computer-generated outcome data enhanced the skills of inexperienced practitioners, and may lead to better surgical performance in the operating theatre. ${ }^{6}$ Several haptic devices are also being used to good effect in dental training, a field in which more traditional simulation models have not been popular because they lack tactile feedback. The so-called 'visio-haptic integrated training simulation system' allows participants to probe and prepare cavities, and uses models of the biomechanical properties of various dental constituents, anatomical structures and dental instruments to provide haptic feedback to the user. It was rated highly by dentists in terms of usability (how useful the system was for improving their hands-on experience), clarity (of haptic feedback from the system), effectiveness (how easily the user could achieve what they wanted to accomplish) and satisfaction (how engaging the system was to use). ${ }^{1}$

The acquisition of the necessary hands-on skills involving physical interaction with patients (e.g. anatomical manipulation, surgical procedures) by health care professionals (HCPs) is a time-consuming task that has traditionally been based on close supervision by an experienced mentor or trainer, with or without the use of models. Moreover, as the time allotted to training is ever shrinking due to work pressure and restricted funding streams, computer-aided virtual reality medical training offers a promising addition to help HCPs familiarise themselves with the equipment and procedures, and gain confidence in the acquisition of skills in advance of actual patient encounters.

Current training in intrauterine techniques usually involves using a 'plastic model', such as a 'Zoe'. By their nature, these models offer limited potential to recreate the natural anatomical variations that are encountered in real life. The real training experience is gained in the clinical situation, where not only does the trainee 


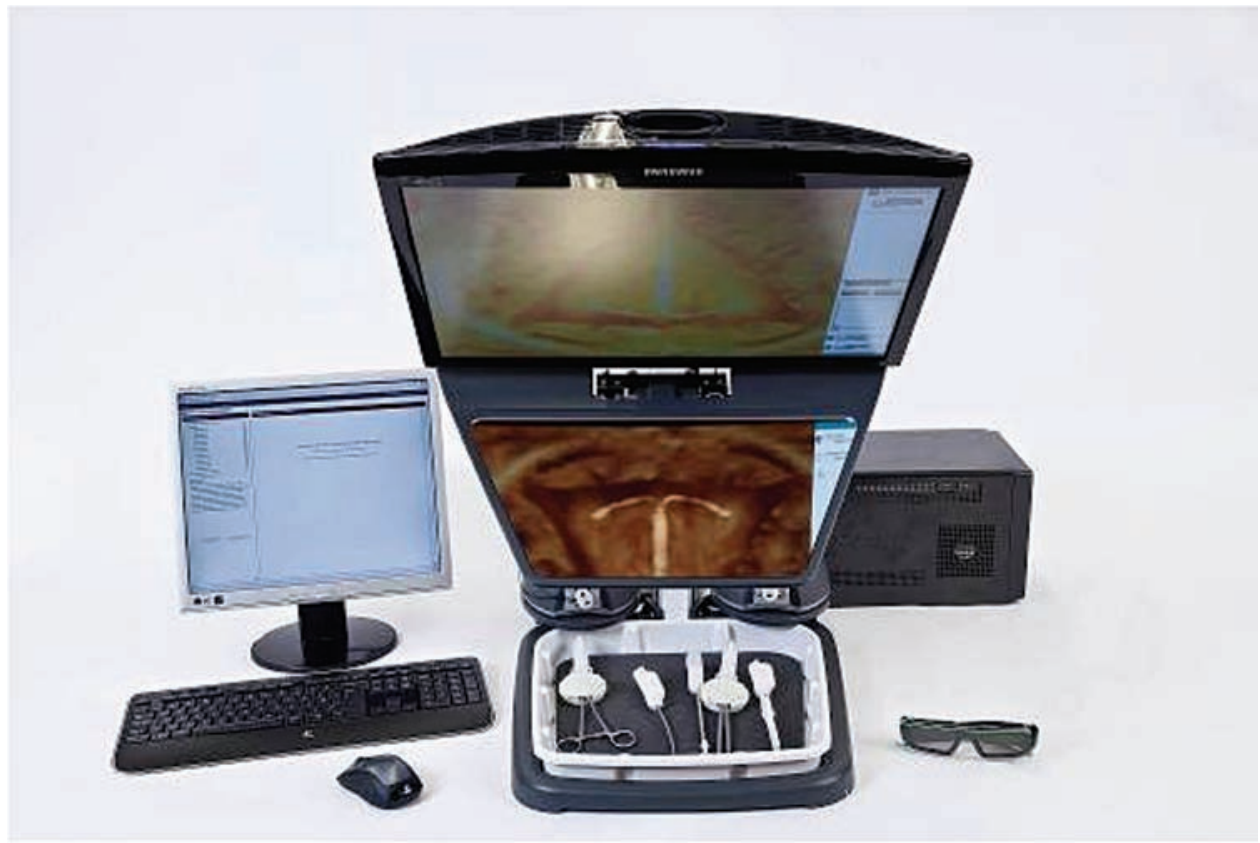

Figure 1 The Mirena ${ }^{\circledR}$ Haptic intrauterine system placement simulator. @ Bayer HealthCare Pharmaceuticals. Image reproduced with the kind permission of Bayer HealthCare Pharmaceuticals.

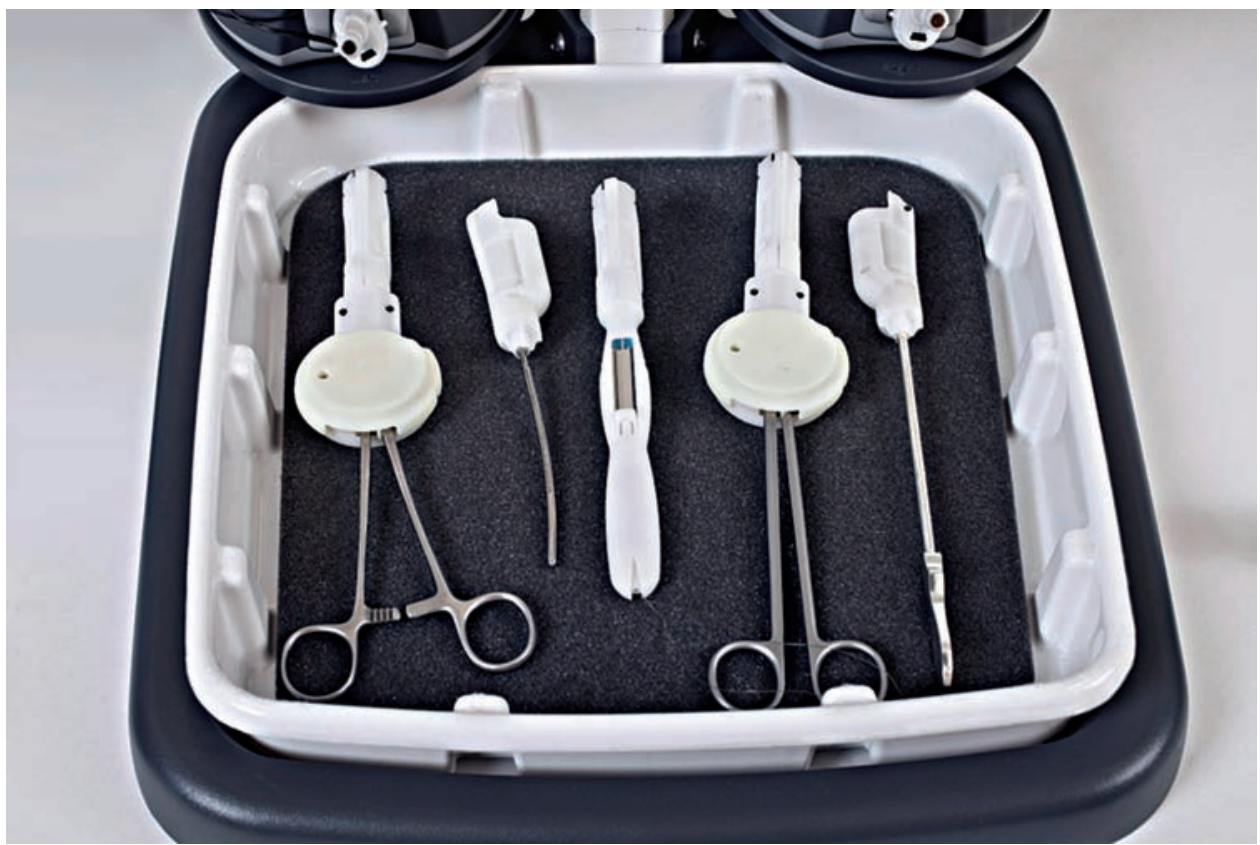

Figure 2 Examples of Mirena ${ }^{\circledR}$ Haptic 'end effectors'. (C) Bayer HealthCare Pharmaceuticals. Image reproduced with the kind permission of Bayer HealthCare Pharmaceuticals.

have to deal with the procedure itself, but he or she is also affected by the reaction of the patient, who is often anxious and will be aware that the inserter is a trainee. Haptic intrauterine system (IUS) training could be used to reduce the number of times the trainer has to intervene or duplicate procedures (e.g. sounding and device position), which can only improve the experience for the patient.

\section{Mirena ${ }^{\circledR}$ Haptic}

Based on this marriage of technologies for the visual and tactile senses, Bayer HealthCare Pharmaceuticals in combination with UK Haptics, Newcastle, UK is developing and designing a Mirena ${ }^{\circledR}$ Haptic model that will allow the user to practise placement of the contraceptive IUS, Mirena. UK Haptics Limited is developing the Haptic program; the software is termed the 
Dr Anne Connolly: an IUT trainer's perspective

- I feel strongly that it is important to promote long-acting reversible contraception methods, and that this strategy is highly dependent on the availability of competent and confident clinicians trained in intrauterine techniques (IUT) and subdermal implant insertion. As a Faculty-registered trainer I have been helping doctors and nurses to gain their Letter of Competence in IUT for many years. I enjoy teaching and I find helping someone to acquire a new skill very rewarding; but, as any trainer will acknowledge, supervising the initial procedures undertaken by any trainee is a stressful experience for all, including the most experienced trainers.

- I think the Mirena ${ }^{\circledR}$ Haptic is an exciting development for this application, bringing IUT training into the 21st century and having significant advantages over the models and devices currently used. I have practised on this simulator and found it to be very realistic.

- I expect that this training tool will make it easier for trainees to become competent in the techniques required and to gain experience in some of the technical difficulties routinely encountered. This should increase their confidence when doing the first few initial procedures.

- Mirena Haptic will be ideal for use when training in group theory sessions; as an additional session attached to the Diploma theory training; at university-led nurse training days; or for doctors who are updating their skills for re-accreditation. I also believe there is potential for this to be developed further to become a tool for training for other gynaecological procedures such as hysteroscopic surgery and colposcopy.

'Clinical Skills Trainer' or CST. CST provides access to the virtual reality simulator, which will allow users (HCPs) to develop their clinical skills in a virtual environment. The assumption is that users will be gaining Mirena placement confidence and experience.

Mirena Haptic is the first placement simulator and differs from other medical procedure simulators in several ways. The three-dimensional images are of sufficient quality to create a realistic visual virtual environment. The user views the simulated placement procedure on a three-dimensional screen using three-dimensional glasses (Figure 1), much the same as viewing three-dimensional films in the cinema. The haptic device is fitted with 'end effectors' that look and feel like the instruments used for IUS placement (i.e. tenaculum, metal sound, scissors and Mirena inserter) (Figure 2). The simulator reacts in real time to every movement the user makes, and provides feedback during the procedure on the progress made.

There are currently 12 patient scenarios from which the HCP can choose to practise: these include parous and nulliparous women, anteverted, retroverted, anteflexed or retroflexed uteri, and women with cervical stenosis requiring dilation. The HCP can view the human anatomy as well as feel it using the end effectors. Each end effector movement provides tactile feedback so all interactions with the anatomy can provide a feeling of resistance similar to the expected sensation in real life. Preparatory steps that would be required in the clinic (e.g. pelvic examination) need to be considered in the correct order, prior to proceeding with insertion. Completion of the insertion procedure tasks is performed in the same way and in the same sequence as it would be during an actual patient encounter. There is also an opportunity to obtain an ultrasound image and check the position of the device after insertion.

Mirena Haptic is designed to be split into component parts so that they fit into small reinforced suitcases, enabling them to be easily transported. It will be available in many countries including the UK, Germany, France, Poland, Australia, Brazil, Canada and the Asia Pacific region. The system is available in English, Spanish, German, French and Portuguese. All models in all languages will have the capability of both hardware and software upgrades.

Acknowledgements The authors would like to thank UK Haptics (www.ukhaptics.co.uk) for their collaboration in the development of the Mirena ${ }^{\circledR}$ Haptic module in conjunction with Bayer HealthCare Pharmaceuticals. The authors would also like to thank inScience Communications, a Wolters Kluwer business, for editorial assistance with the development of this article.

Funding Funding was provided by Bayer HealthCare Pharmaceuticals.

Competing interests Dr Anne Connolly has received consultancy fees from Bayer HealthCare Pharmaceuticals, the manufacturer of Mirena ${ }^{\circledR}$. Dr Sarah Rybowski is an employee of Bayer HealthCare Pharmaceuticals.

Provenance and peer review Not commissioned; externally peer reviewed.

\section{References}

1 Konukseven EI, Onder ME, Mumcuoglu E, et al. Development of a visio-haptic integrated dental training simulation system. J Dent Educ 2010;74:880-891.

2 Pan JJ, Chang J, Yang X, et al. Graphic and haptic simulation system for virtual laparoscopic rectum surgery. Int J Med Robot 2011;7:304-317.

3 Fluet GG, Qiu Q, Kelly D, et al. Interfacing a haptic robotic system with complex virtual environments to treat impaired upper extremity motor function in children with cerebral palsy. Dev Neurorehabil 2010; 13:335-345.

4 Miyoshi T, Takahashi Y, Lee H, et al. Upper limb neurorehabilitation in patients with stroke using haptic device system: reciprocal bi-articular muscle activities reflect as a result of improved circle-drawing smoothness. Disabil Rehabil Assist Technol 2010; $5: 370-375$.

5 Froelich JM, Milbrandt JC, Novicoff WM, et al. Surgical simulators and hip fractures: a role in residency training? J Surg Educ 2011;68:298-302.

6 Modi CS, Morris G, Mukherjee R. Computer-simulation training for knee and shoulder arthroscopic surgery. Arthroscopy 2010;26:832-840. 